\title{
A new definition of postconcussive syndrome
}

\author{
E. Sander Connolly Jr., MD, ${ }^{1}$ and Richard G. Ellenbogen, MD² \\ 'Department of Neurosurgery, Neurological Institute of Columbia University, New York, New York; and 2Department of \\ Neurological Surgery, University of Washington, Seattle, Washington
}

$\mathrm{T}$ ATOR and colleagues present data on 284 consecutive patients, referred over 16 years, who were diagnosed with a concussion. Of these 284 patients, 221 (an extremely high $78 \%$ of the patients) were diagnosed with postconcussion syndrome (PCS). ${ }^{1}$ The PCS diagnosis was based on a newly refined definition that includes persistence of at least 3 symptoms for more than a month. The principal aim of this study was to redefine the term PCS based on a more detailed review of the authors' experience. The average age of the patients was 27 years old and two-thirds of the PCS cases resulted from sports and recreation, with a median symptom duration of 7 months. While the average number of concussions suffered in those with PCS was 3, nearly a quarter of patients had suffered just a single concussion. Consistent with previous studies, preexisting comorbidities were associated with an increased risk of prolonged recovery from concussion. A quarter of the patients in this study with PCS also had a past history of psychiatric disorder, attention-deficit hyperactivity disorder, learning disability, or migraine. The average number of symptoms was 8 in the PCS group and more symptoms were noted in females, those involved in litigation, those with extracranial injuries, and those with a history of loss of consciousness and/or amnesia. Not surprisingly, and consistent with previous studies, more symptoms predicted a longer duration of PCS, and more previous concussions was associated with more symptoms. A wide range of medical specialists evaluates patients after a concussion including pediatrics, family medicine, emergency medicine, and sports medicine. The very high rate of PCS in this select referral population likely reflects the purely neurosurgical referral bias, as $85 \%-90 \%$ of patients in most sports-related concussion series will recover spontaneously in the first week after injury. Additional evidence in support of this being a highly select and perhaps unique population is the higher incidence of arachnoid cysts and Chiari Type I malformations than in an unselected population. This likely resulted from evaluation by the authors of challenging patients who are sent to an experienced neurological surgery practice specializing in posttraumatic brain-injured patients to determine the risk for "return to play" medical decisions. The significant conclusions from this paper warrant attention. The authors redefine the term PCS based on a specific population consisting of those unlikely to spontaneously recover in the first month after injury. This rational, albeit arbitrary, definition of PCS provides us with insights into the rather heterogeneous population of concussed active patients most likely to be referred to neurological surgeons. This is not a pure population of young or elite athletes, but instead more reflective of a general active population including both sexes and a wide range of activities and ages. The authors wisely conclude that the use of the International Classification of Diseases, 10th Revision (ICD-10), and Diagnostic and Statistical Manual of Mental Disorders, 4th Edition (DSM-IV), to define PCS in a general population of active patients involved in recreational activity is entirely inadequate, as the authors have identified the more severe manifestations of PCS. Neither the patients' postinjury symptomatology nor their recovery is as benign as previously believed, and the predictors for delayed recovery have been appropriately broadened based on this study.

http://thejns.org/doi/abs/10.3171/2015.7.JNS151335

\section{References}

1. Tator CH, Davis HS, Dufort PA, Tartaglia MC, Davis KD, Ebraheem A, et al: Postconcussion syndrome: demographics and predictors in 221 patients. J Neurosurg [epub ahead of print February 26, 2016. DOI: 10.3171/2015.6.JNS15664]

\section{Disclosures}

The authors report no conflict of interest. 


\section{Response}

\section{Charles H. Tator, MD, PhD, FRCSC}

Department of Neurosurgery, Toronto Western Hospital, Toronto, Ontario, Canada

We are grateful to the editors of the Journal of Neurosurgery for deeming our article on postconcussion syndrome (PCS) worthy of an editorial, and we are also grateful to the authors of the editorial, E. Sander Connolly Jr. and Richard G. Ellenbogen, for their insightful comments. As they correctly indicated, the PCS cases sent to a neurosurgeon for management are highly selected and could be expected to differ from the cases referred to other health care professionals, and the neurosurgical cases are likely more severe, with a greater number of symptoms, and longer lasting. Nevertheless, the editorial writers concluded that information in the article is useful to other practitioners seeing patients with PCS. The editorial writers also agree with the authors' views about the inadequacy of previous definitions of PCS in the ICD-10 and the DSM-IV. It was also gratifying that the editorial writers confirmed the authors' views that PCS is not as benign a condition as previously believed. The editorial writers were also favorable toward the authors' broadening of the list of predictors of delayed recovery. 